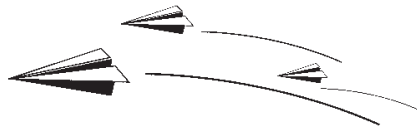

Erhalt der tierischen Insuline in der Schweiz

Der Rückzug von Lente ${ }^{\circledR}$ MC durch Novo Nordisk bringt das Thema der tierischen Insuline wieder ins Gespräch. Die Schweizerische Diabetes-Gesellschaft, Novo Nordisk Pharma AG und CP Pharma (Schweiz) AG setzen sich auch weiterhin für den Erhalt der tierischen Insuline in der Schweiz ein. Den realen Möglichkeiten ist dabei Rechnung zu tragen.

Die Bedeutung der tierischen Insuline ist unbestritten: Für eine engagierte Gruppe von Diabetes-Betroffenen stellen sie ein Bedürfnis dar. Deshalb setzt sich die Schweizerische Diabetes-Gesellschaft SDG seit Jahren für den Erhalt der tierischen Insuline in der Schweiz ein. Dabei wurden beachtliche Erfolge erzielt. So sind mit Novo Nordisk Pharma und mit CP Pharma in der Schweiz immer noch zwei Anbieter vertreten. Das Insulin Lente ${ }^{\circledR}$, welches auf den Weltmärkten nicht mehr erhältlich ist, wurde bisher noch in Kasachstan und in der Schweiz vertrieben.

Die Anzahl der Diabetes-Betroffenen, die mit einem tierischen Insulin behandelt werden, ist seit längerer Zeit rückläufig. Seit 1997 beträgt der Rückgang $50 \%$, so dass heute aufgrund von Schätzungen von Novo Nordisk noch etwa 440 Betroffene mit Lente ${ }^{\circledR}$ MC behandelt werden. Novo Nordisk hat nun angekündigt, das Insulin Lente ${ }^{\circledR} \mathrm{MC}$ per 31. Oktober 2003 aus dem Handel zu nehmen. Lente ${ }^{\circledR} \mathrm{MC}$ ist ein Verzögerungsinsulin, das $\mathrm{zu} 70 \%$ aus kristallinem Rinderinsulin und $\mathrm{zu} 30 \%$ aus amorphem Schweineinsulin besteht.

Um eine Umstellung auf ein anderes Insulin zu unterstützen, versendet Novo Nordisk ein Informationsschreiben an 6000 bis 7000 Ärztinnen und Ärzte, Diabetesberaterinnen und andere medizinische Fachpersonen in der Schweiz. Die betreffende Information wird auch über den Grosshandel verbreitet. Ziel dieser Aktion ist, konkrete und klare Richtlinien für Umstellungen auf ein anderes Insulin zur Verfügung zu stellen.

In Zusammenarbeit mit der Schweizerischen Diabetes-Gesellschaft setzt sich Novo Nordisk auch künftig für den Erhalt der tierischen Insuline in der Schweiz ein. Novo Nordisk hat sich dazu verpflichtet, bei künftigen Rückzügen tierischer Insuline frühzeitig - wenn immer möglich 1,5 bis 2 Jahre im voraus - zu informieren. Ebenfalls wurde uns zugesichert, dass tierisches Insulin (Actrapid ${ }^{\circledR}$ MC suis) in
Penampullen (für Novo-Pen 3) weiterhin abgefüllt über die Geno-Apotheken bezogen werden kann.

Die in der Schweiz nach dem Rückzug von Lente erhältlichen tierischen Insuline sind allesamt Schweineinsuline. Novo Nordisk bietet ab 31. Oktober 2003 noch vier tierische Insuline an (Actrapid ${ }^{\circledR} \mathrm{MC}$, Insulatard ${ }^{\circledR} \mathrm{MC}$, Mixtard ${ }^{\circledR} 30$ MC und Semilente ${ }^{\circledR}$ MC). Drei weitere tierische Insuline werden durch CP Pharma angeboten (Hypurin ${ }^{\circledR}$ porcine neutral, Hypurin ${ }^{\circledR}$ porcine 30/70 Mix, Hypurin ${ }^{\circledR}$ porcine isophane).

Als Patientenorganisation handelt die SDG nach dem Grundsatz, dass Diabetes-Betroffene möglichst jenes Insulin erhalten, das ihnen am besten zusagt. Die SDG muss jedoch zur Kenntnis nehmen, dass die Umsätze für tierische Insuline rückläufig sind. Als Patientenorganisation wird sie sich nach wie vor nicht an diesbezüglichen medizinischen Diskussionen beteiligen. Sie wird sich jedoch weiterhin im Rahmen des Möglichen für den Erhalt der tierischen Insuline in der Schweiz einsetzen.

Schweizerische Diabetes-Gesellschaft 


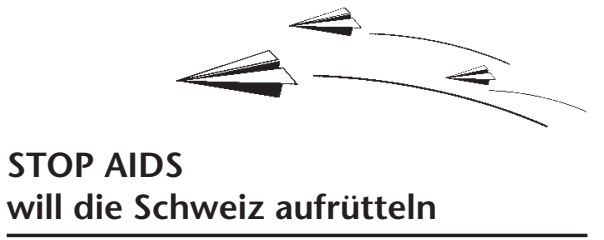

Das BAG lanciert die Kampagne 2003

Das Bundesamt für Gesundheit (BAG) lanciert in Zusammenarbeit mit der Aids-Hilfe Schweiz die STOP-AIDS-Kampagne 2003. Ab 22. April werden in der ganzen Schweiz 7000 Plakate mit Slogans auf gelbem Hintergrund ausgehängt. Die neue Kampagne fordert eine ganze Reihe von «Autoritäten» auf, sich für die Prävention zu engagieren. Aids soll wieder zu einem zentralen Thema der Öffentlichkeit werden.

$\mathrm{Ab}$ 22. April werden die Plakate der STOPAIDS-Kampagne 2003 so ziemlich überall in der Schweiz zu sehen sein: kurze, zum Teil ironische Texte auf stechend gelbem Hintergrund. Die Slogans - 87 deutsche, 40 französische und 25 italienische - sollen bei Leserinnen und Lesern Reaktionen hervorrufen, indem sie sich an verschiedene «Autoritäten», Institutionen, Gruppierungen, Geschäfte oder Akteure des gesellschaftlichen Lebens wenden. Die Slogans sind auf klare Botschaften reduziert und wollen für die HIV/Aids-Prävention einen günstigen Boden vorbereiten. Eine weitere Neuheit: die rund 7000 Plakate der Kampagne 2003 werden nicht nach dem Zufallsprinzip im öffentlichen Raum ausgehängt, sondern sozusagen in ihrem «natürlichen» Umfeld plaziert. So wird etwa ein Slogan zum Sextourismus an Flughäfen zu finden sein.

\section{Zwei Phasen}

Zwei unterschiedliche Plakatierungsphasen bilden die Kampagne. In der ersten Phase ab 22. April 2003 werden die Plakate für eine Dauer von vier Wochen in der ganzen Schweiz zu sehen sein. Das Zielpublikum dieser ersten Serie ist die allgemeine Bevölkerung. Einige Slogans werden von Multiplikatoren verkündet, das heisst von Instanzen, die durch ihre Nähe zu gewissen Bevölkerungskreisen geeignet sind, dem Präventionsaufruf eine grössere Wirkung zu verleihen. Open Air Festivals, Grossverteiler, Lehrer- und Elternschaft sind beispielsweise solche Partner.

Die zweite Phase der STOP-AIDS-Kampagne ist für den August geplant; sie wird am 12. August beginnen und ebenfalls vier Wochen dauern. Sie vermittelt Botschaften der direkten Prävention und wendet sich an bestimmte Zielgruppen wie die Gay Community oder Migranten/-innen, die von der Zunahme der Neuinfektionen im Jahr 2002 besonders betroffen waren.

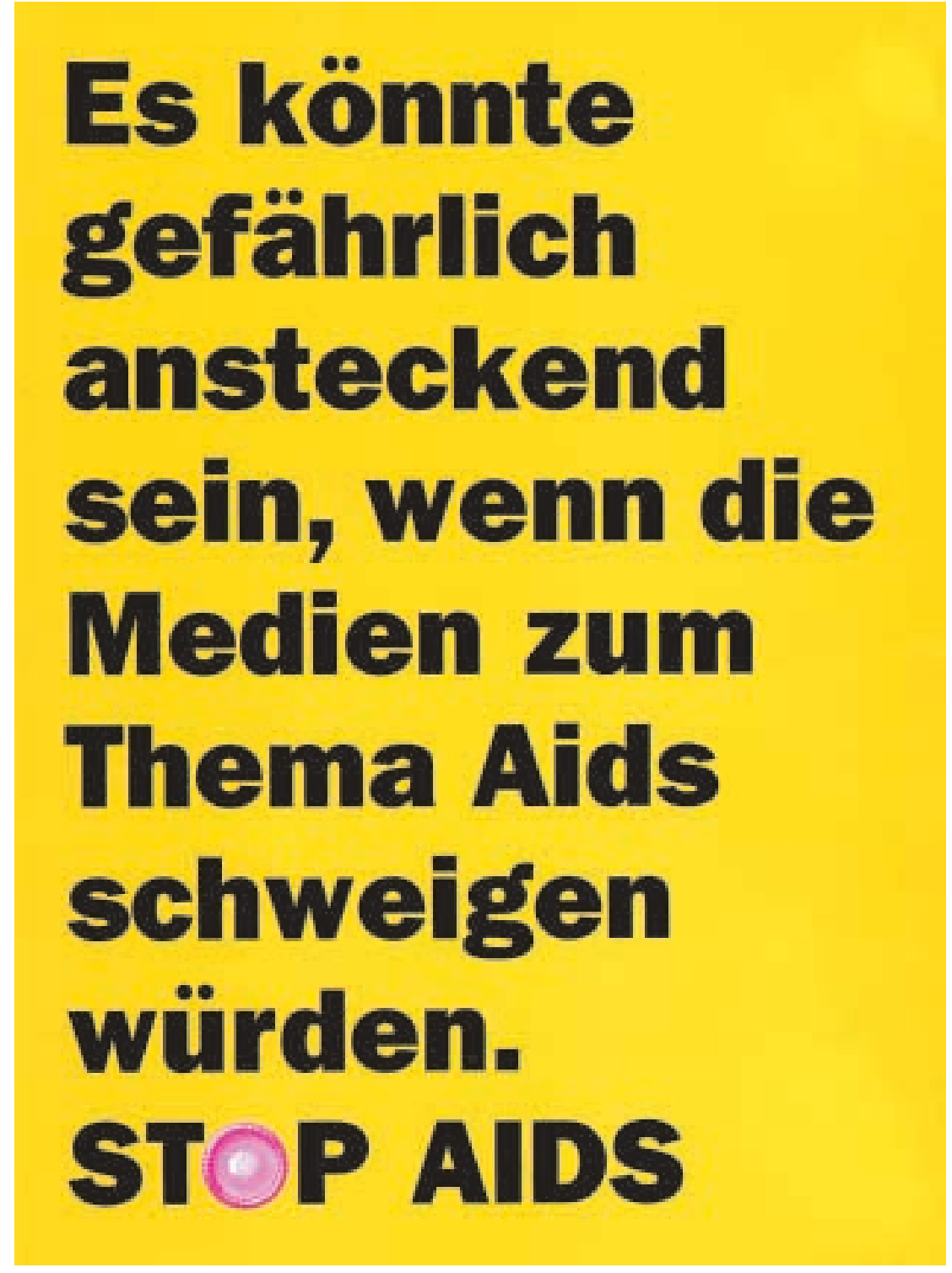

Weshalb eine neue Kampagne?

War die Anzahl der neu diagnostizierten HIVInfektionen zwischen 1992 und 2000 rückläufig, so nahm diese Zahl im Jahr 2001 leicht zu $(7,7 \%)$ und belief sich auf 631 Fälle. Der Aufwärtstrend bestätigte sich im folgenden Jahr mit einer Zunahme um 25,5\% (+161 Fälle) auf insgesamt 792 Fälle. Allein die jährlichen medizinischen Kosten für die Behandlung der 161 Fälle übersteigen in einigen Jahren das Budget der STOP-AIDS-Kampagne 2003 (Fr. 3 Mio.).

Die meisten der diagnostizierten Neuinfektionen aus dem Jahr 2002 wurden auf sexuellem Weg übertragen. Die stärkste Zunahme (+37\%) an neuen HIV-Fällen wurde bei Männern, die mit Männern Sex haben (men having sex with men, MSM) beobachtet; es handelt sich dabei hauptsächlich um Schweizer. Aber auch bei den Heterosexuellen war die Zunahme markant: sie betrugt $26,2 \%$ und betraf vor allem Staatsangehörige von Ländern südlich der Sahara.

\section{Spezialmassnahmen}

Angesichts der epidemiologischen Situation wendet sich das Bundesamt für Gesundheit parallel zu den zwei Plakatphasen der STOPAIDS-Kampagne 2003 zusätzlich mit spezifischen Massnahmen an MSM. Lokale, wo sexuelle Aktivitäten möglich sind und «vor Ort» praktiziert werden - Saunen, Bars, Discos, Klubs mit Darkrooms - sollen motiviert werden, ihre Gäste in geeigneter Form zu informieren.

Die Aids-Hilfe Schweiz als Co-Absenderin der Kampagne unterstreicht, dass mit der Propagierung des Präservativs die Präventionsarbeit keineswegs erledigt sei. Daneben braucht es das Engagement in der Beratung, wo die Ängste, die Wünsche und die Sorgen der Individuen zur Sprache kommen können. 